\title{
Motor Branch Biopsy of the Pronator Teres Muscle in a Patient with Painful Forearm Neuropathy
}

\author{
Tomomi Kinoshita $^{a} \quad K^{2}$ azuhiro Fukushima ${ }^{c}$ Ryu-ta Abe ${ }^{a}$ Yuka Ogawa \\ Michitaka Nakagawa ${ }^{a}$ Nagaaki Katoh $^{a}$ Takuhiro Yoshida ${ }^{a}$ \\ Hiroyuki Kato $^{b}$ Shu-ichi Ikeda ${ }^{a}$ \\ Departments of ${ }^{a}$ Medicine (Neurology and Rheumatology) and ${ }^{b}$ Orthopedic Surgery, \\ Shinshu University School of Medicine, and 'Intractable Disease Care Center, Shinshu \\ University Hospital, Shinshu University School of Medicine, Matsumoto, Japan
}

\section{Key Words}

Motor nerve branch biopsy - Multiple mononeuropathy - Muscle biopsy · Nerve biopsy · Painful neuropathy · Pronator teres

\begin{abstract}
Histological evaluation of a peripheral nerve is often the final diagnostic work-up for a neuropathy of unknown origin, and a distal sensory nerve is usually biopsied. Here, we report the case of a female patient with painful unilateral neuropathy in the upper arm. According to the histological evaluation of the pronator teres motor branch, vasculitis seemed to be the most probable cause of the condition, and steroid therapy improved the patients' symptoms. A biopsy of the motor branch of the pronator teres muscle nerve may be considered a valuable diagnostic option in selected cases with neuropathy affecting the upper limb, when performed in cooperation with neurologists and orthopedic surgeons. @ 2014 S. Karger AG, Basel
\end{abstract}

\section{Introduction}

Histological evaluation of a peripheral nerve is often the final diagnostic work-up for a neuropathy of unknown origin, and is most informative when the nerve is clinically affected [1]. A distal sensory nerve, including the sural, superficial peroneal, or superficial radial nerve, is usually biopsied, and a motor nerve branch is rarely considered [2-4]. Here, we report a case with painful unilateral neuropathy in the upper arm. In this case, a biopsy of a

Kazuhiro Fukushima

Intractable Disease Care Center, Shinshu University Hospital

Shinshu University School of Medicine

3-1-1 Asahi, Matsumoto 390-8621 (Japan)

E-Mail kfuku@shinshu-u.ac.jp 
Kinoshita et al.: Motor Branch Biopsy of the Pronator Teres Muscle in a Patient with Painful Forearm Neuropathy

pronator teres motor branch provided useful information in consideration of the pathogenesis.

\section{Case Report}

A previously healthy 19-year-old Japanese woman noticed a skin rash on her lower limbs. After 4 months, the rash spread to her hands and forearms. In February 2013 (9 months from the onset of the exanthema), she was admitted to our hospital because of sudden paresis and pain in her left upper limb. At the first presentation, she showed livedo reticularis in all four limbs, with a temperature of $36.5^{\circ} \mathrm{C}$, a pulse of 65 beats per minute, and a blood pressure of $112 / 62 \mathrm{~mm} \mathrm{Hg}$. Examination showed decreased sensation in the radial side of her left forearm and dorsum of the hand. Manual muscle testing was uncompleted in her left forearm because of severe neuralgia, although it was normal in the left proximal arm and lower limbs.

Laboratory findings were normal for sedimentation rate $(8.0 \mathrm{~mm} / \mathrm{h})$, leukocyte counts $\left(5,880 / \mathrm{mm}^{3}\right)$, serum C-reactive protein $(0.01 \mathrm{mg} / \mathrm{dl})$, serum creatinine $(0.49 \mathrm{mg} / \mathrm{dl})$, and urinalysis. Tests for serum antibodies, including antinuclear, anti-DNA, anti-RNP, anti-SSA and anti-SSB, IgG anticardiolipin, and anti-beta-2-glycoprotein I, were negative. Lupus anticoagulant was negative. Serum antineutrophil cytoplasmic antibody was negative in both perinuclear and cytoplasmic staining patterns, and antimyeloperoxidase and proteinase 3 specificity were not detected. Thyroid function was normal, while antithyroid peroxidase and antithyroglobulin antibodies were positive. The results of the serological tests for acute infection with Epstein-Barr virus, herpes simplex virus, varicella-zoster virus, cytomegalovirus, human parvovirus B19, and Mycoplasma pneumoniae were all negative. Antibodies to human immunodeficiency virus (HIV) and hepatitis B and $C$ viruses were also negative. Cerebrospinal fluid analysis showed a normal cell count of $1 / \mathrm{mm}^{3}$ (mononuclear cells) with normal total protein concentration $(26 \mathrm{mg} / \mathrm{dl}$, normal $<40 \mathrm{mg} / \mathrm{dl})$. MRI scans of the brain, whole spine, left upper limb, cervical nerve roots, and brachial plexus and wholebody computed tomography scans showed no abnormal findings. Skin biopsy specimens from the livedo reticularis in her left lower limb showed no specific findings of vasculitis or thrombophlebitis.

In neurophysiological studies, the patient's motor nerve conduction in the median, ulnar, and radial nerves was within the normal range; however, the compound muscle action potential and conduction velocity in the left radial nerve $(8.4 \mathrm{mV}$, normal $>7.0 \mathrm{mV} ; 64.4 \mathrm{~m} / \mathrm{s}$, normal $>60 \mathrm{~m} / \mathrm{s}$, respectively) were decreased in comparison with the right radial nerve (10.6 mV and $91 \mathrm{~m} / \mathrm{s}$, respectively). The result of the sensory nerve conduction study was normal. Whilst we were unable to record the motor unit potentials during voluntary contraction of the left forearm muscles because of the patient's severe pain, needle electromyography showed fibrillation potentials in her left extensor carpi radialis muscle. Ultrasound examination of the ulnar, median, and radial nerve in the left forearm showed no abnormalities.

Livedo reticularis and severe neuralgia pointed to vasculitic neuropathy as the most likely pathological condition in this patient. Due to the acute progression of the motor impairment, a single course of intravenous immunoglobulin $(400 \mathrm{mg} / \mathrm{kg}$ body weight daily for 5 consecutive days) with methylprednisolone pulse therapy (intravenous methylprednisolone $1 \mathrm{~g}$ daily for 3 consecutive days) was commenced. Improvement of the patient's neuralgic pain and motor dysfunctions was observed within a few days of commencement of the therapy. However, severe tenderness along the median nerve in her left forearm and 
muscle weakness mainly in the left median nerve territory remained. Manual muscle testing showed muscle weakness in the left median nerve territory: flexor digitorum profundus (second finger: $3 / 5$; third finger: $4 / 5$; intact in fourth and fifth finger), flexor digitorum superficialis muscle (second finger: $2 / 5$; third finger: $2 / 5$; fourth finger: $4 / 5$; intact in fifth finger), and pronator teres muscle (2/5). A second MRI of the left upper limb showed new abnormal signal intensities in the median nerve at the forearm and muscles innervated by the radial and median nerve, including flexor pollicis longus, flexor digitorum profundus, flexor digitorum superficialis, extensor carpi ulnaris, and extensor digitorum (fig. 1). These findings were suggestive of the presence of mononeuropathy multiplex involving the left median and radial nerves (predominant in the former).

Despite vasculitic neuropathy being the most likely causative pathological condition in this patient, histological confirmation was required in order to justify the length of her treatment with potentially cytotoxic medications. A superficial radial nerve biopsy was not encouraged because there were no apparent findings of its involvement. We performed a combined biopsy of the left pronator teres muscle and a motor branch of the median nerve to the pronator teres. The procedures were performed in the operating room under conduction anesthesia. We found that her left pronator teres motor nerve had two branches, one proximal branch going to the superficial (humeral) head and the other distal branch going to the deep (ulnar) head of the pronator teres muscle. We biopsied the latter, which was a very thin nerve branch, to reduce potential motor complications of the procedure. There were no complications during or after the biopsy.

In our case, the specimens from a motor branch, that contained only one nerve bundle, showed severe loss of myelinated fibers with a sectional distribution, myelin ovoids with many foamy macrophages, and marked edema in the perineurium and subperineurium (fig. $2 \mathrm{~A})$. The specimens from the pronator teres muscle showed typical neurogenic features including small group atrophy with small-angulated muscle fibers (fig. 2B). These findings indicated ischemic nerve injury. As expected, vasculitis seemed to be the most probable cause of the condition, although direct findings (e.g., fibrinoid necrosis of wall vessels) were not seen in these specimens.

As a result of these histological findings, two cycles of a 3-day regimen of high-dose intravenous methylprednisolone $(1 \mathrm{~g} /$ day $)$ were initiated, followed by oral prednisolone at $0.5 \mathrm{mg} / \mathrm{kg} /$ day. The patient's neuralgia remitted and muscle weakness was gradually alleviated. The patient was discharged in April 2013, and returned to full-time housekeeping with no notable neurological deficits. She is currently continuing treatment with low-dose oral prednisolone under active follow-up.

\section{Discussion}

The original technique of diagnostic biopsy of the pronator teres and a motor branch of the median nerve was described at academic meetings [5, 6] and reported in a retrospective review of 20 patients as a safe and useful procedure to differentiate between motor neuropathy and motor neuron disease in patients with weakness of the upper limbs [4]. A biopsy of the motor branch of the pronator teres muscle nerve may be considered a valuable diagnostic option in selected cases with neuropathy affecting the upper limb, when performed in cooperation with neurologists and orthopedic surgeons. 
Kinoshita et al:: Motor Branch Biopsy of the Pronator Teres Muscle in a Patient with Painful Forearm Neuropathy

\section{Acknowledgements}

This work was supported by a Health and Labour Sciences Research Grant on Intractable Diseases (Pathogenesis and Diagnostic Accuracy of Neuropathic Pain, 23170301 to S.I.) from the Ministry of Public Health, Labour, and Welfare, Japan. We thank Dr. Hiroshi Morita, Dr. Akiyo Hinone, Dr. Daigo Miyazaki, and Dr. Kiyoshiro Nagamatsu (Department of Medicine, Neurology, and Rheumatology, Shinshu University School of Medicine) for neurophysiological and pathological evaluation.

\section{Disclosure Statement}

The authors have no conflicts of interest to disclose.

\section{References}

1 Said G, Lacroix C: Primary and secondary vasculitic neuropathy. J Neurol 2005;252:633-641.

2 Agadi JB, Raghav G, Mahadevan A, Shankar SK: Usefulness of superficial peroneal nerve peroneus brevis muscle biopsy in the diagnosis of vasculitic neuropathy. J Clin Neurosci 2005;19:1392-1396.

-3 Collins MP, Mendell JR, Periquet MI, Sahenk Z, Amato AA, Gronseth GS, Barohn RJ, Jackson CE, Kissel JT: Superficial peroneal nerve/peroneus brevis muscle biopsy in vasculitic neuropathy. Neurology 2000;55:636-643.

4 Dy CJ, Lange DJ, Jones KJ, Garg R, DiCarlo EF: Diagnostic biopsy of the pronator teres and a motor branch of the median nerve: indications and technique. J Hand Surg Am 2012;37:2570-2575.

5 Chan N, Ting J, Lange DJ: Biopsy of the median motor nerve: description of technique and complications. Ann Neurol 2007;62:S60.

6 Addona T, Ting J: Diagnostic Biopsy of Median Motor Nerve Branch: Indications, Technique, and Applications. Presented at the New York Regional Society of Plastic Surgeons Residents' Night. New York, 2007. 


\section{Case Reports in Neurology}

\begin{tabular}{l|l}
\hline Case Rep Neurol 2014;6:202-206 \\
\hline DOI: 10.1159/000365883 & $\begin{array}{l}\text { C 2014 S. Karger AG, Basel } \\
\text { www.karger.com/crn }\end{array}$ \\
\hline
\end{tabular}

Kinoshita et al.: Motor Branch Biopsy of the Pronator Teres Muscle in a Patient with Painful Forearm Neuropathy

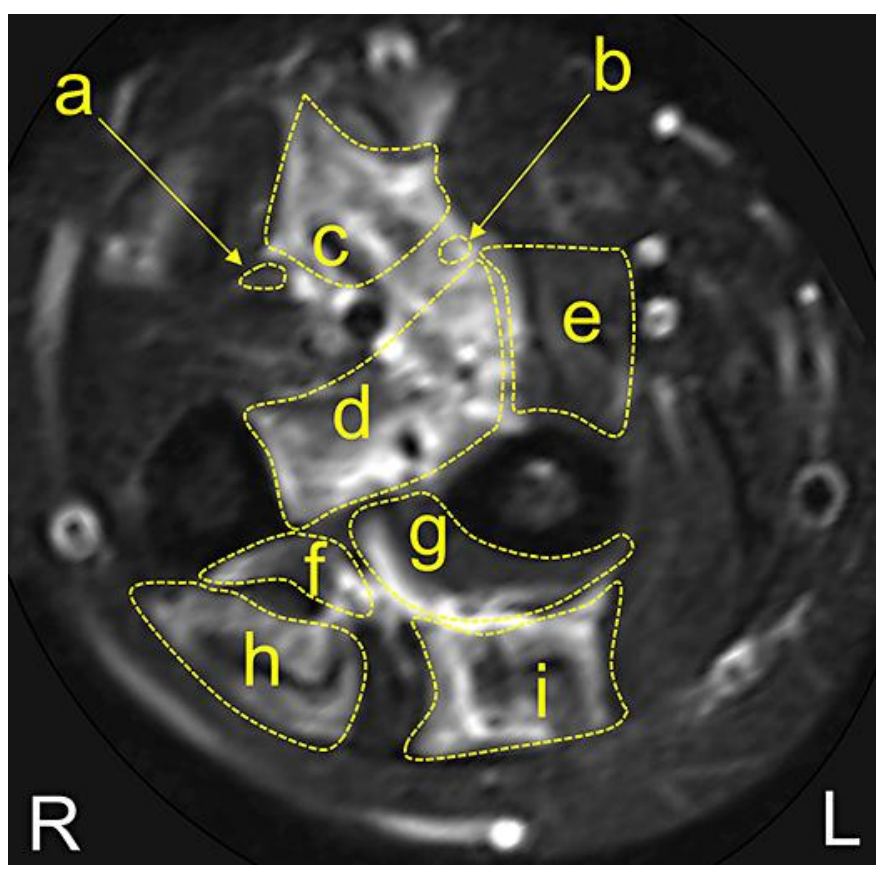

Fig. 1. Axial short TI inversion recovery (1.5T, TR 7,300 ms, TE $71 \mathrm{~ms}$ ) MRI of the left forearm. Median nerve (b), flexor digitorum superficialis (c), flexor digitorum profundus (d), pronator teres (e), extensor pollicis longus (f), spinator (g), extensor carpi ulnaris (h), and extensor digitorum (i) showed abnormal high signal intensity. The ulnar nerve (a) showed normal signal intensity.
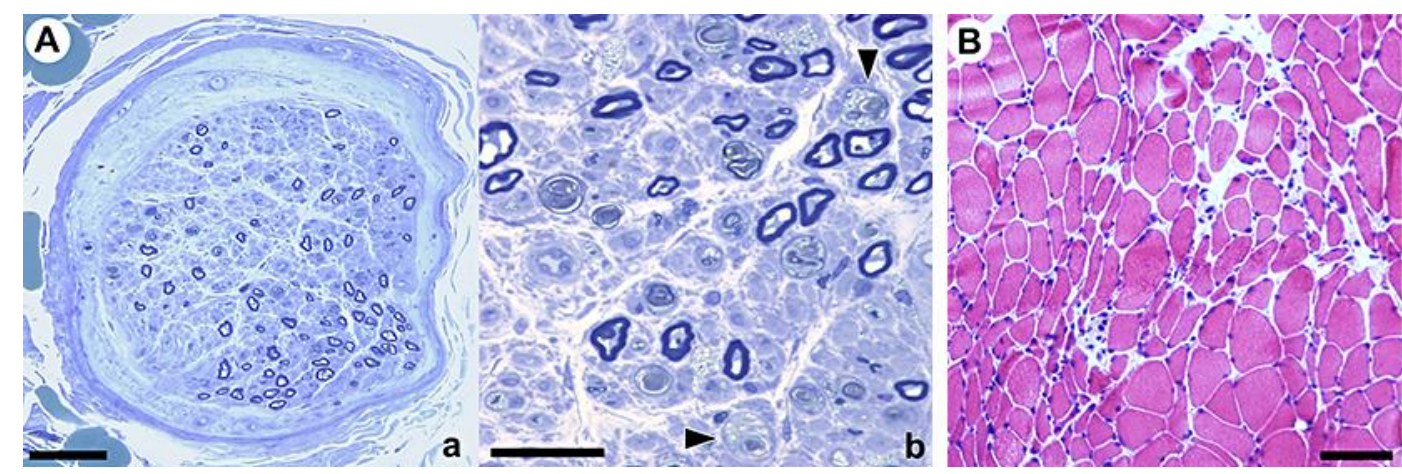

Fig. 2. Specimens from a motor branch (a single nerve bundle) of the pronator teres muscle (toluidine blue) disclosed sectional loss of myelinated fibers and marked edema in the perineurium and subperineu$\operatorname{rium}(\mathbf{A}-\mathrm{a}, \mathrm{bar}=100 \mu \mathrm{m})$. At higher magnification, severe loss of myelinated fibers with a sectional distribution and foamy macrophages (arrowhead) are visible (A-b, bar $=50 \mu \mathrm{m}$ ). The specimens from the pronator teres muscle showed typical neurogenic features including small group atrophy with small angulated muscle fibers (B, bar $=100 \mu \mathrm{m}$ ). Direct findings of vasculitis (e.g., fibrinoid necrosis of wall vessels) or thrombosis were not observed in these specimens. 